\title{
Correction to: Preventing emergency department visits among patients with cancer: a scoping review
}

\section{Scott W. Kirkland ${ }^{1} \cdot$ Miriam Garrido-Clua $^{1}$ • Daniela R. Junqueira ${ }^{1} \cdot$ Sandra Campbell $^{2} \cdot$ Brian H. Rowe $^{1,3}$}

Published online: 24 June 2020

(C) Springer-Verlag GmbH Germany, part of Springer Nature 2020

\section{Correction to: Support Care Cancer https://doi.org/10.1007/s00520-020-05490-1}

Table 2 is incorrect in the original manuscript. The correct table 2 is shown below

The online version of the original article can be found at https://doi.org/ $10.1007 / \mathrm{s} 00520-020-05490-1$

$\triangle$ Brian H. Rowe

brian.rowe@ualberta.ca

1 Department of Emergency Medicine, Faculty of Medicine \&Dentistry, University of Alberta, 1G1.43WMC 8440-112 Street NW, Edmonton, AB T6G 2B7, Canada

2 J.W. Scott Health Sciences Library, University of Alberta, 2K3.28 Walter C. Mackenzie Health Sciences Centre 8440-112 Street NW, Edmonton, AB T6G 2R7, Canada

3 School of Public Health, University of Alberta, Edmonton, AB, Canada 
Table 2 Characteristics of the interventions according to activities implemented and health care provider involvement

Study Patient education Symptom monitoring Patient management Patient Navigator Telephone follow-up

\begin{tabular}{|c|c|c|c|c|c|c|}
\hline \multicolumn{7}{|l|}{ Hospital at Home } \\
\hline Aibar 2013 [10] & & & & & & Not specified \\
\hline \multicolumn{7}{|l|}{ Patient Navigator } \\
\hline Colligan 2017 [11] & + & & & + & & Non-clinical professional \\
\hline \multicolumn{7}{|l|}{ Inpatient management } \\
\hline Kim 2011 [12] & + & + & + & & + & $\begin{array}{l}\text { Oncology clinical nurse } \\
\text { specialist }\end{array}$ \\
\hline Siew 2017 [13] & & & & + & & Nurse \\
\hline Wang 2014 [14] & & & + & + & & Multidisciplinary team \\
\hline \multicolumn{7}{|l|}{ Early symptom detection } \\
\hline Barbera 2015 [15] & & + & + & & & Clinical team \\
\hline Basch 2016 [16] & & + & + & & & Nurse \\
\hline Howell 2017 [17] & & + & + & & & Not specified \\
\hline Siefert $2015[18]$ & + & + & & & & Clinical team \\
\hline Taamhoej 2018 [19] & & + & & & & Nurse/physician \\
\hline \multicolumn{7}{|c|}{ Routine and symptom-based patient follow-up } \\
\hline Bishop 2014 [20] & + & $+\bullet$ & & + & & Nurse \\
\hline Hoverman 2014 [21] & + & + & + & & + & Oncology-certified nurse \\
\hline Kurtz 2006 [22] & + & + & + & & $+*$ & Nurse \\
\hline Lai 2018 [23] & + & $+\bullet$ & + & + & + & Nurse \\
\hline Martinez 2015 [24] & + & & & & $+*$ & Nurse \\
\hline Salmany 2018 [25] & & $+\bullet$ & + & & + & Pharmacist \\
\hline Shaw 2016 [26] & + & & & + & + & Clinical psychologist \\
\hline Suzuki 2014 [27] & + & $+\bullet$ & & & & Pharmacist \\
\hline \multicolumn{7}{|c|}{ Oncology outpatient clinics } \\
\hline Antonuzzo 2017 [28] & & & + & & & Multidisciplinary team \\
\hline Colligan 2017 [11] & & & + & & + & Multidisciplinary team \\
\hline Burdett 2016 [29] & & & + & & & Nurse practitioner \\
\hline Diyar 2018 [30] & & & + & & & Nurse \\
\hline Harr 2015 [31] & & & + & & & Advanced practice nurse \\
\hline Sivendran 2016 [32] & & & + & & & Nurse/medical oncologist \\
\hline Terzo 2017 [33] & & $+\bullet$ & + & & & Nurse \\
\hline Waters 2019 [34] & & & + & & + & Multidisciplinary team \\
\hline
\end{tabular}

Note: $+=$ component present in strategy; grey represent active component of the interventions; $*$ Telephone and in-person follow-up, $\bullet$ self-reported unstructured symptom assessment

Publisher's note Springer Nature remains neutral with regard to jurisdictional claims in published maps and institutional affiliations. 